\title{
ITINERANT THEORY OF EXCHANGE CONSTANTS \\ IN DILUTED Co-BASED SEMIMAGNETIC SEMICONDUCTORS
}

\author{
J. MAŠEK \\ Institute of Physics, Czecho-Slovak Academy of Sciences \\ Na Slovance 2, 18040 Prague 8, Czecho-Slovakia
}

\begin{abstract}
The exchange constants in diluted semimagnetic semiconductors with Co are much larger than in the Mn doped systems. To explain this, we use a semiquantitative, tight binding version of the coherent potential approach and calculate the electronic structure of II-VI mixed semiconductors containing Co and $\mathrm{Mn}$. The exchange constants are deduced from energies of spin reversal processes. The presence of the Co $3 d$ states at the top of the valence band leads to the observed enhancement of the exchange coupling.
\end{abstract}

PACS numbers: 75.10.Lp, 75.30.Ef, 75.50.Pp

\section{Introduction}

The substitution of cobalt in the cationic sublattice of II-VI compound semiconductors results in a new class of semimagnetic semiconductors which have been investigated recently. The measurements of magnetic susceptibility [1, 2] and inelastic neutron scattering [3] showed that exchange constants in the Co-based SMSC are three to four times larger than the exchange constants in traditional semimagnetic semiconductors containing $\mathrm{Mn}$. This result seems to be surprising at the first glance, and calls for a theoretical explanation [1].

In a recent report [4] I suggested an explanation based on the itinerant theory of magnetic interactions in $\mathrm{Zn}_{1-x} \mathrm{Co}_{x}$ Se. According to these preliminary calculations, two minority-spin Co $3 d$ states are degenerate with the valence band and hybridize therefore with the band states stronger than in the case of $\mathrm{Mn}$. The resulting magnetic polarization of the valence band leads to an enhancement of the coupling between magnetic moments of the Co ions. 
The theoretical prediction that the Co $d$-states contribute to the valence band top was supported by recent photoemission experiments for $\mathrm{Cd}_{1-x} \mathrm{Co}_{x} \mathrm{Se}[5$, 6 ]. This leads us to a more systematic semiquantitative study of the electronic structure and magnetic interactions in $\mathrm{Zn}_{1-x} \mathrm{Co}_{x} \mathrm{Se}$ and $\mathrm{Cd}_{1-x} \mathrm{Co}_{x} \mathrm{Se}$, including a comparison with their Mn-based counterparts.

\section{Single-site theory for exchange constants in mixed crystals}

Classically, the magnetic interactions in the semimagnetic semiconductors are described by a spin Hamiltonian

$$
H=-J_{d d} \sum_{j} S_{i} S_{j}-\frac{1}{2} J_{p d} \sum_{i} S_{i} s(i) .
$$

The summation runs over the cationic sites occupied by a transition metal (TM) ions. $S_{i}$ are the classical spins of the ions, $s(i)$ is the spin density of the (valence) band electrons at the site $i . J_{d d}$ is an exchange constant between the local magnetic moments at neighbouring cation sites, the exchange constant $J_{p d}$ characterizes a local coupling between the moments and valence electrons.

The exchange constants $J_{p d}$ and $J_{d d}$ are related to the energies corresponding to spin reversal processes. If we consider a (hypothetical) ferromagnetic crystal, then $J_{p d}$ defines the energy needed for a spin flip of one valence band electron. The mean-field solution, relevant in the mixed crystal case, reads $\Delta E_{p d}=x J_{p d} S$, where $x$ is the concentration of the magnetic ions, and $S$ is the size of their spins.

Similarly, $J_{d d}$ defines the energy needed to reverse just one local moment in the ferromagnetic phase of the mixed crystal. The mean-field solution to the Heisenberg part of the Hamiltonian (1) is

$$
\Delta E_{d d}=2 x Z J_{d d} S(S+1)
$$

with $Z=12$. (number of the nearest-neighbours in the cationic sublattice).

The relations between the spin-flip energies and the exchange constants give a possibility to calculate $J_{p d}$ and $J_{d d}$ from the electronic structure of the mixed crystal. It is evident that $\Delta E_{p d}$ is the separation of the valence bands for spin-up and spin-down electrons. As usual [7], we identify $\Delta E_{p d}$ with the spin splitting of the valence band top $\left(\Gamma_{15}\right)$ which is well defined also in the case of disordered mixed crystals.

To calculate the other characteristic energy $\Delta E_{d d}$ we assume, in the frame of the static version of the fluctuation theory [8], that the size and direction of the local magnetic moment are given by the local exchange potential. This means that all local exchange potentials are the same in the ferromagnetic phase of the mixed crystal, and that the reversal of the magnetic moment at one site is represented just by changing the polarity of the exchange potential at that site. $\Delta E_{d d}$ is then the change of the total electronic energy due to such local "impurity" in otherwise perfect ferromagnetic arrangement. It can be evaluated by integrating the induced change $\delta g(E)$ of the total density of elecronic states [4],

$$
\Delta E_{d d}=\int_{-\infty}^{E_{\mathrm{F}}} \delta g(E) \mathrm{d} E .
$$




\section{Tight binding model}

The interacting electrons are described by a multisite Anderson Hamiltonian $H=H_{\text {band }}+H_{d}+H_{\text {hybr. }}$. Its three parts correspond to the band states of the host II-VI semiconductor, to the interacting electrons in the $d$-shells of the TM ions, and to the hybridization of the $d$-states with the bands.

The band-structure part is represented by the $s p^{3}$ bands with parametrized transfer integrals [9]. For simplicity, we assume a zinc-blende structure also for CdSe. The hybridization of the $d$-states with the anion states is parametrized according to the Harrison rules with $\eta_{s d \sigma}=-3.75, \eta_{p d \sigma}=-3.50, \eta_{p d \pi}=1.62[10]$.

The interaction part of the Hamiltonian is, for each TM site,

$$
\begin{aligned}
H_{d}= & \sum_{\mu, \sigma} \varepsilon_{\mu} N_{\mu, \sigma}+[(U+J) / 2] \sum_{\mu, \sigma} N_{\mu, \sigma} N_{\mu,-\sigma} \\
& +(U / 2) \sum_{\mu, \nu, \sigma} N_{\mu, \sigma} N_{\nu,-\sigma}+[(U-J) / 2] \sum_{\mu, \nu, \sigma} N_{\mu, \sigma} N_{\nu, \sigma} .
\end{aligned}
$$

Here we suppress the site index $i$. The symbols $\varepsilon_{\mu}$ denote theatomic levels (including the crystal field splittings), $U$ and $J$ are the intra-site Coulomb and exchange integrals, $N_{\mu, \sigma}$ are the occupation number operators. We parametrize these quantities according to the self-consistent atomic calculations so that $\varepsilon_{d}(\mathrm{Co})=$ $\varepsilon_{d}(\mathrm{Mn})-1.9 \mathrm{eV}, U(\mathrm{Co})=3.3 \mathrm{eV}, J(\mathrm{Co})=0.9 \mathrm{eV}$, and adjust $\varepsilon_{d}(\mathrm{Mn})$ so that the occupied Mn $d$-states in CdSe lie at binding energies $-3.1 \mathrm{eV}$.

\section{Spectral distribution of the $d$-states and the exchange constants}

Basic information about the distribution of the transition metal $d$-states in the bands of the semimagnetic mixed crystals is obtained already from a single impurity calculations. We use the unrestricted Hartree-Fock approximation to calculate the effective atomic levels classified by spin and symmetry $\left(t_{2}\right.$ and $\left.e\right)$. The resulting orbital occupation numbers for CdSe:Mn and CdSe:Co are very close to 0 or 1 , and both $\mathrm{Mn}$ and Co impurities have a high-spin configuration with magnetic moments $2.44 \mu_{\mathrm{B}}$ and $1.43 \mu_{\mathrm{B}}$, respectively. In the Co $3 d^{7}$ shell, both spin-up and spin-down states with the symmetry $e$ are occupied so that the exchange splitting of these states is much smaller than in the case of $d_{t}$ states. This has two important consequencies for the spectral distribution of the Co $3 d$ states: (i) two minority-spin spin $d_{e \downarrow}$ states lie just at the valence band top; (ii) the majority-spin $d_{e \uparrow}$ states at cca $-3.5 \mathrm{eV}$ lie above the $d_{e \downarrow}$ states even in the tetrahedral crystal field.

The densities of states calculated by the coherent potential method for the mixed crystals retain both of these features $[6,4]$.

The position of the $d$-levels is important for the kinetic exchange coupling which is mediated by the spin polarization of the valence band. In the case of $J_{p d}$ only the distance of the $d_{t}$-states from the valence band top is relevant. It decreases from Mn- to Co-based systems and this is reflected by an increase of antiferromagnetic $J_{p d}\left(-1.40 \mathrm{eV}\right.$ in $\mathrm{Cd}_{0.9} \mathrm{Co}_{0.1} \mathrm{Se}$ and $-1.42 \mathrm{eV}$ in $\mathrm{Zn}_{0.9} \mathrm{Co}_{0.1} \mathrm{Se}$ vs. $-1.04 \mathrm{eV}$ in $\mathrm{Cd}_{0.9} \mathrm{Mn}_{0.1} \mathrm{Se}$ and $-0.99 \mathrm{eV}$ in $\mathrm{Zn}_{0.9} \mathrm{Mn}_{0.1} \mathrm{Se}$ ). 
The enhancement of the exchange constant $J_{d d}(\mathrm{Co}-\mathrm{Co})$ with respect to $J_{d d}(\mathrm{Mn}-\mathrm{Mn})$ is even larger $\left(-4.5 \mathrm{meV}\right.$ in $\mathrm{Cd}_{0.9} \mathrm{Co}_{0.1} \mathrm{Se}$ and $-4.65 \mathrm{meV}$ in $\mathrm{Zn}_{0.9} \mathrm{Co}_{0.1} \mathrm{Se}$ vs. $-1.4 \mathrm{meV}$ in $\mathrm{Cd}_{0.9} \mathrm{Mn}_{0.1} \mathrm{Se}$ and $-1.16 \mathrm{meV}$ in $\mathrm{Zn}_{0.9} \mathrm{Mn}_{0.1} \mathrm{Se}$ ). The reason for this is that also the states with symmetry $e$, which are very close to the valence band states, participate in the hybridization effects mediating the kinetic exchange between two local moments.

The calculated exchange constants $J_{p d}$ and $J_{d d}$ are both in a reasonable agreement with the experimental data $[11,12,1,2]$. A quantitative theory based on $a b$ initio calculations has to dispose of the problem of the exchange potential in the Co $3 d^{7}$ shells.

\section{References}

[1] A. Lewicki, A.I. Schindler, J.K. Furdyna, W. Giriat, Phys. Rev. B 40, 2379 (1989).

[2] A. Lewicki, A.I. Schindler, I. Miotkowski, J.K. Furdyna, Phys. Rev. B 41, 4653 (1990).

[3] T.M. Giebultowicz, J.J. Rhyne, J.K. Furdyna, P. Klosowski, J. Appl. Phys. 67, 5096 (1990).

[4] J. Mašek, Solid State Commun. 78, 351 (1991).

[5] K. Kopalko, B.J. Kowalski, B.A. Orłowski, A. Mycielski, V. Cháb, Acta Phys. Pol. A77, 403 (1990).

[6] V: Cháb, J. Mašek, B.A. Orłowski, B.J. Kowalski, Acta Phys. Pol. A 80, (1991).

[7] J.A. Gaj, J. Ginter, R.R. Gałązka, Phys. Status Solidi B 89, 655 (1978).

[8] T. Moriya, Spin Fluctuations in Itinerant Electron Magnetism, Springer, Berlin 1985.

[9] Yu. Li, P.J. Lin-Chung, Phys. Status Solidi B 153, 215 (1989).

[10] W.A. Harrison, Electronic Structure and the Properties of Solids, Freeman, San Francisco 1980.

[11] X. Liu, A. Petrou, B.T. Jonker, G.A. Prinz, J.J. Krebs, J. Warnock, Appl. Phys. Lett. 55, 1023 (1989).

[12] M. Nawrocki, F. Hamdani, J.P. Lascaray, Z. Golacki, J. Deportes, Solid State Commun. 77, 111 (1991). 